\title{
Renal safety and osteonecrosis of the jaw in cancer patients receiving intravenous bisphosphonates
}

\author{
Sercan Aksoy · Omer Dizdar · Kadri Altundag
}

Published online: 27 May 2009

(C) Humana Press Inc. 2009

To the Editor,

We read with great interest the article by Bonomi et al. [1] in which they evaluated the renal toxicity and osteonecrosis of jaw (ONJ) in 398 cancer patients treated with bisphosphonates (BP). They found that 16 patients (4\%) developed renal toxicity after a median of 24 months of BP treatment and 10 patients $(2.5 \%)$ were diagnosed as having ONJ after a median of 39 months on BP. Concerning the renal safety of intravenous BP, we also want to give information about our study [2] in which we retrospectively evaluated 43 breast cancer patients with bone metastases who received zoledronic acid (ZA) more than 24 months. We also found no statistically significant differences in the pre- and post-treatment levels of serum creatinine, blood urea nitrogen. Our results also support the current study that patients treated with BP showed low incidence of renal toxicity. Concerning the ONJ, our previous study [3] performed in large series of cancer patients receiving IV BP showed that 16 of 1,338 patients with breast cancer (1.2\%) and 13 of 548 patients with multiple myeloma (2.4\%) developed ONJ. Supporting the current study that the median dose and duration of treatment with pamidronate or zoledronic acid in our study were significantly higher in patients with ONJ. In conclusion, BP clearly provides an important enhancement in the quality of life by reducing skeletal-related events and reducing pain. Osteonecrosis of the jaw and renal toxicities related to BP should be kept in mind especially in cancer patients receiving $\mathrm{BP}$ in long-term period.

\section{References}

1. Bonomi M, Nortilli R, Molino A, Sava T, Santo A, Caldara A, et al.. Renal toxicity and osteonecrosis of the jaw in cancer patients treated with bisphosphonates: a long-term retrospective analysis. Med Oncol. 2009 Mar 28 [Epub ahead of print].

2. Dincer M, Altundag K, Harputluoglu H, Aksoy S, Cengiz M, Gullu I. Renal safety of zoledronic acid administration beyond 24 months in breast cancer patients with bone metastases. Med Oncol. 2008; 25:356-9. doi:10.1007/s12032-008-9045-3.

3. Hoff AO, Toth BB, Altundag K, Johnson MM, Warneke CL, Hu $\mathrm{M}$, et al. Frequency and risk factors associated with osteonecrosis of the jaw in cancer patients treated with intravenous bisphosphonates. J Bone Miner Res. 2008;23:826-36. doi:10.1359/jbmr.080 205.
S. Aksoy · O. Dizdar $\cdot$ K. Altundag $(\bowtie)$

Department of Medical Oncology, Hacettepe University Institute of Oncology, Sihhiye, Ankara 06100, Turkey

e-mail: altundag66@yahoo.com 